\title{
The latency-associated nuclear antigen of Kaposi sarcoma-associated herpesvirus induces B cell hyperplasia and lymphoma
}

\author{
Farnaz D. Fakhari, 1,2 Joseph H. Jeong, ${ }^{3}$ Yogita Kanan, $^{2}$ and Dirk P. Dittmer ${ }^{1}$ \\ 1Department of Microbiology and Immunology and Lineberger Comprehensive Cancer Center, University of North Carolina at Chapel Hill, \\ Chapel Hill, North Carolina, USA. ${ }^{2}$ University of Oklahoma Health Sciences Center, Oklahoma City, Oklahoma, USA. \\ ${ }^{3}$ Dana-Farber Cancer Institute and Harvard Medical School, Boston, Massachusetts, USA.
}

\begin{abstract}
Kaposi sarcoma-associated herpesvirus (KSHV) is a human lymphotropic herpesvirus. It is implicated in $B$ cell neoplasias such as primary effusion lymphoma and multicentric Castleman disease in AIDS patients. The KSHV latency-associated nuclear antigen (LANA) is consistently expressed in all KSHV-associated tumor cells and was shown to bind the tumor suppressor proteins p53 and pRb. To test LANA's contribution to lymphomagenesis in vivo we generated transgenic mice expressing LANA under the control of its own promoter, which is B cell specific. All of the transgenic mice developed splenic follicular hyperplasia due to an expansion of $\mathrm{IgM}^{+} \mathrm{IgD}^{+} \mathrm{B}$ cells and showed increased germinal center formation. We also observed lymphomas, implying that LANA can activate $B$ cells and provide the first step toward lymphomagenesis.
\end{abstract}

\section{Introduction}

Approximately one-fourth to one-third of all human cancers are of viral etiology (1). Kaposi sarcoma-associated herpesvirus (KSHV, also referred to as HHV-8) is a human lymphotropic $(\gamma)$ herpesvirus. It is tightly associated with the development of Kaposi sarcoma (KS) as well as 2 B cell lymphoproliferative diseases: primary effusion lymphoma (PEL) and multicentric Castleman disease (MCD; reviewed in ref. 2). MCD is a follicular hyperplasia of lymphoid tissue and in its more aggressive form has been described as an expansion of plasma cells. Patients with MCD often develop secondary B cell lymphomas as well as $\mathrm{KS}$. PEL is a lymphoma of B cell origin that is characterized by increased CD138/syndecan-1 expression (a plasma cell maker) and lymphomatous effusions $(3,4)$. KSHV has also been found in a subset of solid diffuse large B cell lymphomas in AIDS patients (5-8), implicating this virus in multiple B cell lineage malignancies. Therefore, we hypothesized that KSHV infection renders mature $\mathrm{B}$ cells hyperresponsive to antigen stimulation. Such hyperactive B cells provide an expanded target for secondsite mutations or cytokine-driven hyperplasia, cumulating in lymphoma in the immune suppressed.

Several PEL cell lines have been established in culture, all of which harbor the latent form of KSHV. PELs are monoclonal, have rearranged their IgG loci, and, on the basis of transcriptional profiling, have been characterized as a post germinal center (GC), or plasmablastoid lymphoma $(4,9,10)$. In a systematic attempt to determine the contribution of KSHV viral genes to $\mathrm{KSHV}$-associated cancers, we previously analyzed the transcription pattern of all KSHV mRNAs in KS and PEL cell lines in culture and in xenograft tumors (11-13). The KSHV latency-

Nonstandard abbreviations used: FSC, forward scatter; KLH, keyhole limpet hemocyanin; KS, Kaposi sarcoma; KSHV, Kaposi sarcoma-associated herpesvirus; LANA, latency-associated nuclear antigen; LPD, lymphoproliferative disease; MCD, multicentric Castleman disease; MZ, marginal zone; PCNA, proliferating cell nuclear antigen; PEL, primary effusion lymphoma; PNA, peanut agglutinin. Conflict of interest: The authors have declared that no conflict of interest exists. Citation for this article: J. Clin. Invest. doi:10.1172/JCI26190. associated nuclear antigen (LANA) was present in every single tumor, which was consistent with in situ analyses $(14,15)$. The LANA protein is necessary and sufficient for latent viral episome maintenance (16-18). This, however, is not the only function of LANA. LANA binds to the p53 and pRb tumor suppressor proteins as well as glycogen synthase kinase $3 \beta(19-21)$. It can function as a transcriptional activator of its own viral (22) and cellular gene promoters, which in the aggregate changes STAT-, p53-, and pRb-dependent signaling events (23-25). These molecular observations imply a role for LANA in B cell oncogenesis beyond viral maintenance, but so far have not been explored in vivo. Using transgenic mice that expressed LANA protein in mature B cells, we found that LANA activated mature B cells in the absence of antigen stimulation, which predisposed the animals to lymphoma development.

\section{Results}

No animal model currently exists that recapitulates KSHVdependent B cell lymphomagenesis. We reasoned that investigating LANA's transforming potential in transgenic mice represents a reasonable approximation of PEL and MCD. The transgenic approach allowed us to draw upon the accumulated knowledge of murine B lineage development as well as an abundance of defined phenotypic and molecular markers. We used the viral KSHV LANA promoter (LANAp) to achieve authentic expression levels of LANA protein (Figure 1A). This promoter is hypomethylated in latently infected cells and constitutively active in all KSHV-associated malignancies $(11,13,26)$. Furthermore, the LANAp fragment extending to -1299 was active in CD $19^{+}$B cells, but not $\mathrm{CD}^{+} \mathrm{T}$ cells, in the spleen and bone marrow of transgenic mice (27). In an effort to mimic the multicistronic nature of the KSHV latency locus, the transgene construct also contained the GFP gene under control of the thymidine kinase (TK) promoter. However, we could not detect GFP expression (data not shown). Multiple independent founder lines were generated by pronuclear injection and validated by integration-specific Southern blot analysis (Figure 1B). In spleen, transgenic mice 
A

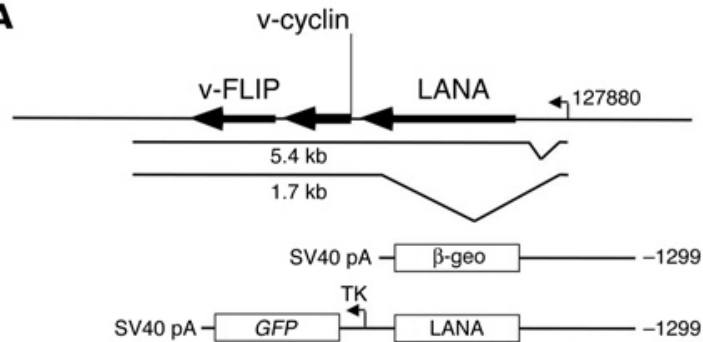

C

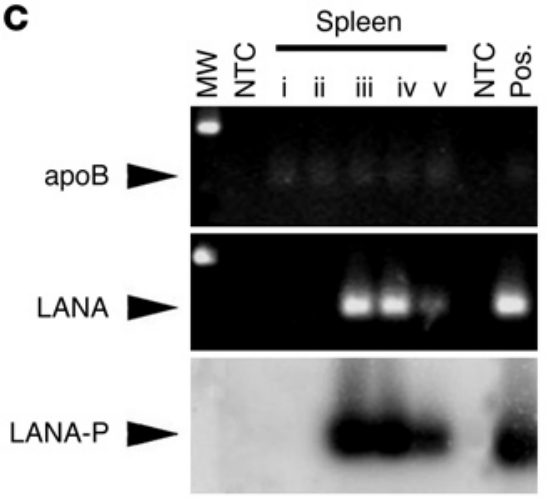

B

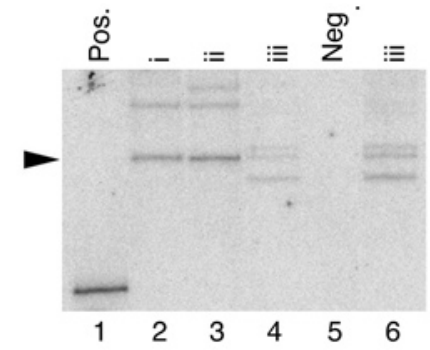

D

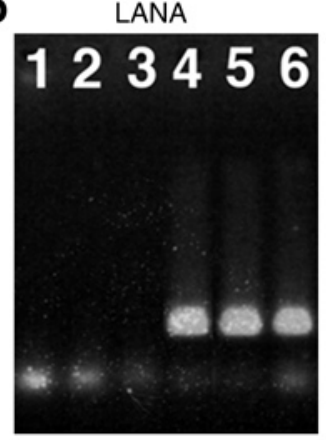

apo

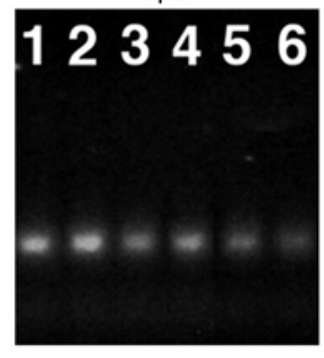

Figure 1

Development of LANA transgenic mice. (A) The LANA transcription locus (14) and transgene construct carrying LacZneo (27) as previously described and the LANA transgene construct used here. SV40 pA, simian virus 40 poly A; $\beta$-geo, $\beta$-galactosidase-neomycin; TK, thymidine kinase. (B) Southern blot analysis of tail DNA, digested with BamHI and hybridized with a LANA-specific probe to reveal the founder-specific integration sites. Lane 1 shows the transgene plasmid as a positive control (Pos.), lane 5 contains the DNA from a C57BL/6 mouse as negative control (Neg.), and remaining lanes represent DNA from LANA transgenic animals belonging to lines i, ii, and iii (animals 4 and 6 are siblings). Arrowhead indicates a fragment specific for all transgenic animals; other bands are integration-site specific. (C and D) Real-time quantitative RT-PCR analysis of spleen samples for $\angle A N A$ transgene mRNA or apoB housekeeping mRNA resolved on a $2 \%$ tris-borate-EDTA-agarose gel and (C) subsequent Southern blot analysis with a probe specific for LANA (LANA-P). Lanes $i-v$ refer to spleen RNA from 5 different transgene-positive animals. NTC, nontemplate control; MW, 100-bp molecular weight marker. (D) Lanes 1-3 show transgene-negative, and lanes 4-6 transgene-positive, littermates after backcross into the C57BL/6 background.

expressed levels of LANA mRNA that were equivalent to those found in PEL as determined by real-time quantitative RT-PCR, which was normalized to murine housekeeping apoB mRNA (Figure 1C). We also detected transgene mRNA in the spleens and kidneys of some animals. However, this did not result in phenotypic changes (data not shown). Consistent with the progressive amplification of LANA-expressing B cells during lymphoid hyperplasia, we observed variation in LANA mRNA levels in the spleens of individual animals. Importantly, the LANA transgene remained transcribed in spleen after 6 generations of backcrosses into a C57BL/6 background (Figure 1D).

To characterize the effect of LANA on B cell development, we examined multiple littermates of 2 independent founder lines in detail. The mice in these experiments were age- and sex-matched littermates and housed together, that is, exposed to similar environmental antigens. To guard against "founder effects," which might skew the phenotype, we started our analysis after the fourth generation of transgenic mice was backcrossed to C57BL/6 mice. Hence, all animals were $(1 / 2)^{4}$ or $\geq 94 \%$ isogenic to their nontransgenic littermates. We detected LANA protein expression within the center of transgenic spleen follicles but not in littermate control animals (Figure 2A). The primary follicles in the LANA transgenic mice were LANA and proliferating cell nuclear antigen (PCNA) positive and larger in diameter (Figure 2B). They were highly proliferative com- pared with control animals as evidenced by PCNA staining. Both LANA and PCNA are located in the nucleus. As B cells have very little cytoplasm, the majority of the signal was nuclear. Hyperplasia was associated with increased spleen weight $(P \leq 0.04$, Student's $t$ test; $n=48$ ) and expanded follicles in $100 \%$ of the transgenic mice. To determine at which stage in B cell development LANA exerted its effect, we surveyed splenic B cell populations using flow cytometry. This revealed a significant increase in forward scatter-high (FSChi; i.e., activated), mature $\mathrm{CD}^{1} 9^{+} \operatorname{IgM}^{+} \operatorname{IgD}^{+} \mathrm{B}$ cells (Table 1). The increase in the $\mathrm{FSC}^{\mathrm{hi}} \operatorname{IgD}^{+} \operatorname{IgM}^{+}$cell population was consistent with follicular hyperplasia and statistically significant $(P<0.0002$, Mann-Whitney U test; $n=26$ ). Early GC and activated follicular cells also were increased $\left(\mathrm{CD} 21^{-} \mathrm{CD} 23^{+} \mathrm{CD} 19^{+} \mathrm{IgD}^{+}\right)$. Marginal zone (MZ) cells (CD21 $\left.{ }^{+} \mathrm{CD} 23^{-} \mathrm{CD} 19^{+} \mathrm{IgD}^{-}\right)$, which differentiate to $\mathrm{GC}$ in response to $\mathrm{T}$ cell-independent antigens, were not affected. Later stages in antigen-dependent $B$ cell maturation such as late GC $\left(\mathrm{CD} 45 \mathrm{R}^{+} \mathrm{CD} 27^{-} \mathrm{CD} 40^{+} \mathrm{CD} 38^{-}\right)$, memory $\left(\mathrm{CD} 45 \mathrm{R}^{+} \mathrm{CD} 27^{+} \mathrm{CD} 40^{-}\right.$ $\left.\mathrm{CD} 38^{+}\right)$, and plasma cells $\left(\mathrm{IgG}^{+} \mathrm{CD} 138^{+}\right)$also were not affected.

To further characterize the LANA-positive population in the LANA transgenic mice, we performed immunohistochemistry. Peanut agglutinin (PNA) and GL-7 are associated with activated GC B cells. IgD is a marker for mature B cells that is lost upon differentiation late during GC maturation. PNA staining revealed marked differences between normal and transgenic animals. All 
A

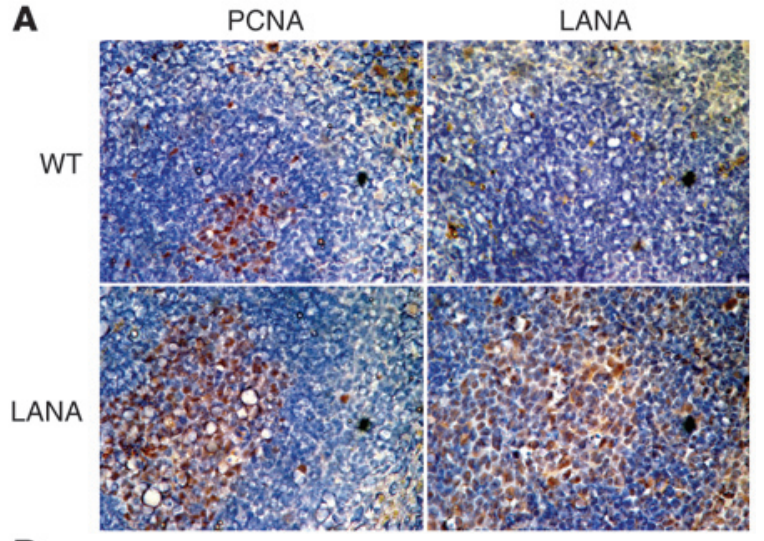

B

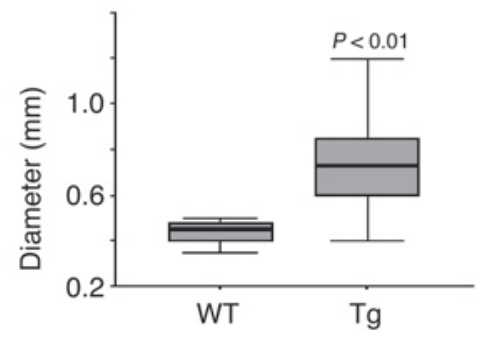

LANA transgenic mice (100\%) presented with enlarged, irregular patches of PNA-positive cells compared with the fewer, smaller PNA-positive foci seen in control mice (Figure 3). In addition, we observed single, extrafollicular PNA-positive cells in the LANA transgenic mice, suggesting that these cells assumed activated characteristics outside the GC context. Consistent with our flow cytometric analysis, IgD remained expressed in the hyperproliferative foci of LANA transgenic mice (Figure 3). Few GL-7-positive foci (i.e., late GC) were present in wild-type mice. By comparison to PNA, the GL-7 positive foci were equally rare and at times smaller in the LANA mice than in wild-type mice (Figure 3). This became particularly evident at higher magnification. The control (no primary antibody) showed no background staining in either wild-type or LANA transgenic mice. We did not observe changes in $\mathrm{T}$ cell distribution as determined by CD3 staining (data not shown).

To examine the impact of antigen exposure on LANA mice, we immunized with NP(30)-keyhole limpet hemocyanin [NP(30)$\mathrm{KLH}]$ and after 7 days stained the spleens again with PNA. As before, LANA transgenic mice, but not wild-type littermates, showed prominent PNA-positive foci in the absence of immunization (Figure 4). With regard to $\mathrm{GC}$ formation we did not detect any difference between salinetreated $(n=8)$ and $\mathrm{NP}(30)$ KLH-immunized $(n=9)$ LANA transgenic mice. In contrast, wild-type mice responded with GC formation to $\mathrm{NP}(30)-\mathrm{KLH}$ $(n=4)$, but not to saline $(n=3)$. This phenotype is

\section{Table 1}

$\mathrm{FL}$, follicular.

\section{Figure 2}

LANA expression in spleens. (A) Immunohistochemistry using a rat $m A b$ against LANA (right panels) or rabbit polyclonal antibody against PCNA (left panels) and visualization with NovaRed (brown) and hematoxylin (blue) counterstain in a transgene-negative and a LANApositive animal. Arrowhead indicates the localization of LANA-positive cells. Magnification, $\times 400$. (B) Diameter of LANA-positive foci for wildtype and transgenic animals $(P \leq 0.01, n=11)$. The box represents the interquartile range, the line within the box indicates the median and whiskers indicate the highest and lowest values.

consistent with LANA activating mature $\mathrm{B}$ cells $\left(\mathrm{PCNA}^{+}, \mathrm{PNA}^{+}\right.$, $\mathrm{FSC}^{\mathrm{hi}}$ ) in the absence of antigen, causing focal aggregation. However, the cells did not complete the GC reaction without antigen and remained $\operatorname{IgD} \mathrm{DLL}^{+} 7^{-}$. At this point we do not know whether in response to KLH immunization the LANA transgenic mice arrested at early GC stage (pre-GL-7) or exhibited an accelerated phenotype in which most of the KLH-responsive B cell clones had already left the GC and migrated to the bone marrow as mature plasma cells.

The tumors in LANA transgenic animals were of B cell lineage. Figure 5 shows an example of a LANA transgenic animal, which at autopsy presented with severe splenomegaly (spleen size greater than 5 times that of the LANA-positive littermate control). Medium-sized plasma cells with abundant cytoplasm and prominent central nuclei and a mixture of immunoblasts and plasmablasts with anaplastic plasmacytoma phenotype (28) were observed on $\mathrm{H} \& \mathrm{E}$ sections. We defined plasma cells according to McHeyzer-Williams and colleagues (29) as CD27-/surface IgG-K ${ }^{+}$for plasma I and B $220^{+} / \mathrm{CD} 138^{+}$for plasma II. Plasma I $(20 \%)$ and plasma II (16\%) cell populations (Figure 5, H and G) were significantly expanded relative to littermate controls $(2 \%$ and 3\%, respectively; Figure 5, J and I). This phenotype in the LANA transgenic mouse model represents an expansion of the same B cell population as KSHV-associated human lymphomas $(9,30)$, most closely resembling MCD and plasmablastic lymphomas (31). In situ analysis showed that major portions of the spleen were taken over by IgG- $\kappa^{+} / \lambda$ - lymphoma cells (Figure 5 , A and C, and Supplemental Figure 1; supplemental material available online with this article; doi:10.1172/JCI26190DS1), while only a few clustered IgG-positive cells were evident in normal mice over 300 days old (Figure 5, B and D) and in normal LANA littermate controls of the same age and sex. The lymphoma

Changes in B cell compartments in LANA transgenic mice

\begin{tabular}{|c|c|c|c|c|c|c|c|c|}
\hline \multirow[b]{2}{*}{ Class } & \multirow[b]{2}{*}{ Marker } & \multicolumn{3}{|c|}{ Wild type } & \multicolumn{3}{|c|}{ LANA } & \multirow[b]{2}{*}{$P$} \\
\hline & & $\%$ & SD & $n$ & $\%$ & SD & $n$ & \\
\hline Mature & $\lg \mathrm{M}^{+} \lg \mathrm{D}+\mathrm{CD} 19+$ & 13.62 & 4.00 & 6 & 20.06 & 6.09 & 26 & 0.05 \\
\hline Activated & $\operatorname{lgM}+\lg \mathrm{D}+\mathrm{CD} 19+\mathrm{FSC}^{\mathrm{hi}}$ & 4.35 & 0.98 & 6 & 7.05 & 1.60 & 26 & 0.0002 \\
\hline MZ & $\lg \mathrm{M}^{+} \lg \mathrm{D}^{-} \mathrm{CD} 19+$ & 6.09 & 2.33 & 6 & 4.84 & 1.70 & 26 & 0.25 \\
\hline$M Z$ & CD21+CD23-CD19+IgD- & 1.16 & 0.70 & 4 & 2.11 & 0.99 & 25 & 0.06 \\
\hline $\mathrm{FL}$ & CD21-CD23+CD19+IgD+ & 3.61 & 4.59 & 4 & 10.30 & 4.56 & 25 & 0.05 \\
\hline GC & CD21-CD23+CD19+IgD- & 0.50 & 0.19 & 4 & 0.52 & 0.27 & 25 & 0.86 \\
\hline GC & $\mathrm{CD} 19+\mathrm{CD} 23+\mathrm{CD} 40+\mathrm{CD} 38-$ & 7.44 & 3.18 & 4 & 4.35 & 3.69 & 23 & 0.15 \\
\hline Memory & $\mathrm{CD} 45 \mathrm{R}^{+} \lg \mathrm{G}^{+} \mathrm{CD} 27^{+}$ & 9.33 & 1.32 & 5 & 8.65 & 3.17 & 25 & 0.45 \\
\hline Plasma & $\mathrm{CD} 45 \mathrm{R}^{+} \mathrm{CD} 27^{-\mathrm{CD} 138^{+}}$ & 0.64 & 0.49 & 6 & 0.53 & 0.24 & 26 & 0.63 \\
\hline
\end{tabular}




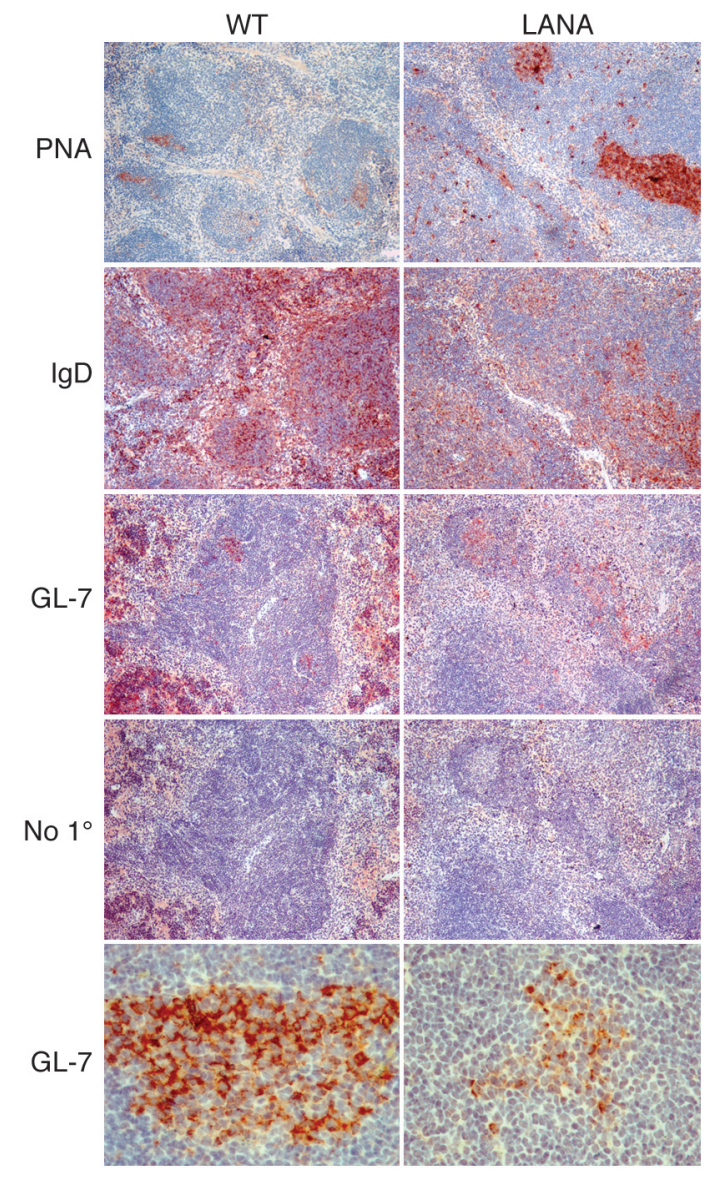

cells were CXC-chemokine receptor 4 positive (CXCR4 $\left.4^{+}\right)$, which on B cells is only expressed at the plasma cell stage (Figure 5, $\mathrm{E}$ and F). All lymphoma cells expressed the LANA protein (Supplemental Figure 2). Based on a review of H\&E-stained spleen sections, $11 \%$ of LANA transgenic mice ( 7 of 63 ) developed lymphomas, twice the rate recorded previously in C57BL/6 mice and in our LANAp-LacZ transgenic mice (32). In addition to plasmacytoma, we observed follicular B cell lymphoma, small lymphocytic lymphoma, and composite lymphoma (Figure 6). We did not observe the exact equivalent to human PEL, since none of the tumors presented as malignant effusions. To differentiate between lymphoproliferative disease (LPD) and lymphoma, we analyzed IgG $V_{H}$ usage in wild-type spleens and LANA transgenic mouse tumors. All tumors were monoclonal, as evidenced by reduction of heterogeneity in the PCR signal, with primers specific for $\mathrm{V}_{\mathrm{H}} 7183: \mathrm{J}_{\mathrm{H}}, \mathrm{Dq} 52: \mathrm{J}_{\mathrm{H}}$, or $\mathrm{V}_{\mathrm{H}} \mathrm{J} 558: \mathrm{J}_{\mathrm{H}}$ family rearrangements each yielding 4 products in wild-type mice (Figure 7). If a tumor was present in the background of normal spleen cells, a nonrandom single band appeared brighter than all the other bands. The specific

\section{Figure 4}

\section{Figure 3}

Analysis of GC development in a representative LANA and littermate wild-type mouse. Markers are indicated on the left (No $1^{\circ}$, no primary antibody). All sections are counterstained blue with hematoxylin. Magnification, $\times 400$ (bottom 2 panels); $\times 100$ (remaining panels).

clonal rearrangement for tumors 2 and 3 was $V_{H} 7183 \mathrm{Dq} 52 \mathrm{~J}_{\mathrm{H}} 2$. For tumor 4 it was $\mathrm{Dq} 52 \mathrm{~J}_{\mathrm{H}} 2$. No $\mathrm{V}_{\mathrm{H}}$ rearrangement was detected for tumor 4, suggesting that the $V_{H}$ rearrangement involved a family other than $V_{H} 7183$ or $V_{H}$ 558. Tumor 1 was an anaplastic plasmacytoma for which clonality was confirmed by monotypic IgG- $\kappa$ light chain expression (Figure 7 and Supplemental Figure 1). Total input DNA was normalized based on real-time quantitative PCR for the single-copy apoB gene, which allowed us to see enrichment of monoclonal tumor cells in the background of some wild-type splenic B cells.

\section{Discussion}

We chose the B cell compartment to query the effects of the KSHV LANA in vivo based on the natural history of this virus, which establishes latency in B cells (33) and is associated with PEL and MCD, 2 B cell hyperproliferative diseases. The fact that the KSHV LANA promoter is active in mice is consistent with exhaustive infectivity studies for this virus (34). In particular, de novo infection of SCID-human chimeric mice showed that given the choice between T cells or B cells, only CD19+ B cells supported indefinite LANA expression and latent maintenance of the KSHV episome (35). Using the LANA transgenic mice as guidance, we speculate that in asymptomatic human carriers, infected resident $\mathrm{B}$ cells $\left(\mathrm{IgM}^{+} \operatorname{IgD}{ }^{+} \mathrm{CD} 19^{+}\right)$remain organresident and sustain KSHV latent persistence. Such a model is consistent with the low frequency of KSHV genome-positive cells in circulating peripheral blood lymphocytes of LANA-seropositive individuals $(36,37)$. By comparison, EBV viral loads in asymptomatic carriers are 10- to 1,000-fold higher than KSHV viral loads in asymptomatic KSHV-infected patients (36). Even though memory B cells are generally considered the most longlived B cell population, mature B cells are long-lived as well. In the mouse spleen, resident follicular cells live up to 4.5 months, or 4 times the time required to reach sexual maturity (38), and
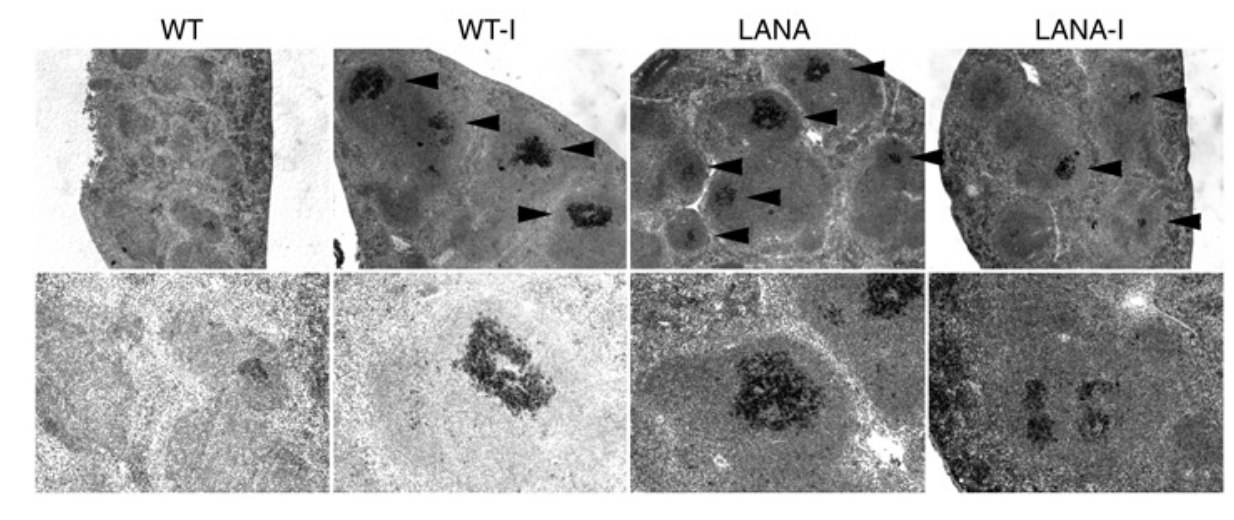

GC response to NP(30)-KLH. Spleen sections of wild-type and LANA transgenic mice with or without NP(30)-KLH immunization (-I) were stained with PNA. Arrowheads indicate GCs. Magnification, $\times 40$ (top panels); $\times 100$ (bottom panels). 


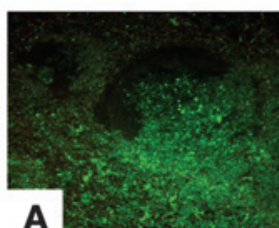

A
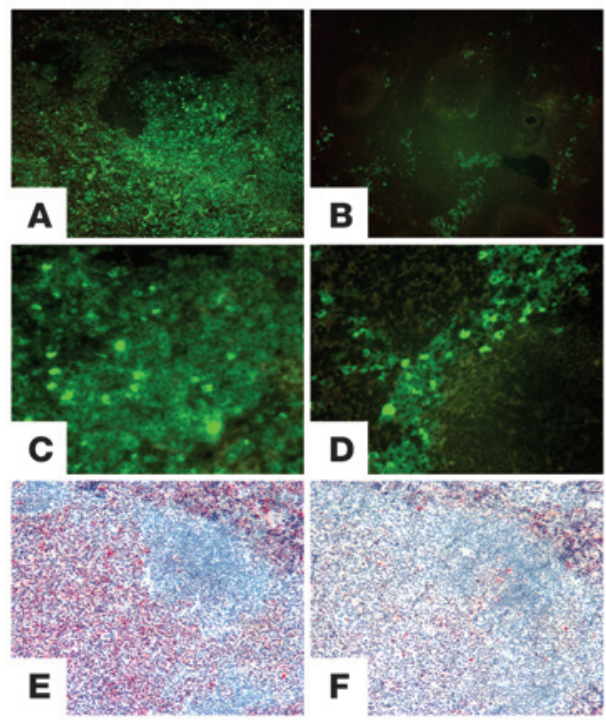

G

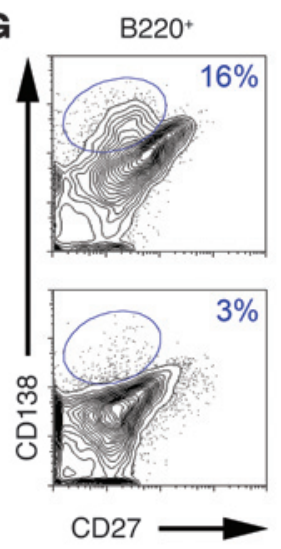

H

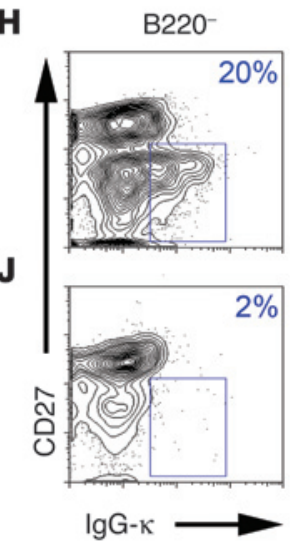

\section{Figure 5}

Anaplastic plasmacytoma in a LANA transgenic animal. (A-D) IgG- $\kappa$ expression (surface and intracellular) in a lymphoma from LANA transgenic ( $\mathbf{A}$ and $\mathbf{C})$ and nontransgenic (B and D) littermates. (E and F) CXCchemokine receptor 4 (CXCR4) for expression in LANA transgenic $(\mathbf{E})$ or control (F) mice. Magnification, $\times 100$ (A, B, E, and F); $\times 400$ (C and D). All sections are counterstained blue with hematoxylin. ( $\mathbf{G}$ and $\mathbf{H}$ ) Flow cytometry analysis of the anaplastic plasmacytoma. (I and $\mathbf{J}$ ) Flow cytometry analysis of spleen of a nontransgenic littermate. ( $G$ and I) Gates represent CD138+B220+CD27- lymphoma cells at $16 \%$ frequency and normal spleen cells at $3 \%$ frequency. ( $\mathbf{H}$ and $\mathbf{J}$ ) Gates represent IgG- $\kappa^{+}$B220-CD27- lymphoma cells at $20 \%$ frequency and nor mal spleen cells at $2 \%$ frequency.

as such represent a stable compartment for lifelong viral persistence. The LANA-dependent hyperactive B cell phenotype uncovered here may induce reactivation/reinfection cycles with high frequency, which were shown to augment latent viral persistence in a KSHV culture model (39).

In the LANA mice, mature B cells were focally aggregated, activated, and hyperproliferative ( $\mathrm{FSC}^{\mathrm{hi}}, \mathrm{PNA}^{+}$, and $\mathrm{PCNA}^{+}$). Typically, this only occurs in response to antigen and constitutes the initial stage in GC formation. Here it resulted in a benign LPD with $100 \%$ penetrance, which increased the chance to sustain a second-site mutation and over time gave rise to frank B cell lymphomas. LANA fostered this developmental step but could not substitute for the paracrine signals and antigen required for complete $\mathrm{GC}$ maturation $\left(\mathrm{IgD}^{-} \mathrm{GL}-7^{+}\right)$. This phenotype is analogous to mice with constitutively active $B$ cell CLL/lymphoma 6 (bcl-6), which also present with LPD, antigen-independent GCs, and low-frequency diffuse large B cell lymphomas at advanced age (40). LANA alone does not transform cells in culture, but ectopic expression in B cell lines rendered cells resistant to p16INK4mediated cell-cycle arrest (25). LANA can also augment cell activation events through the ras/MAPK pathway (21), and this may provide a molecular explanation for how LANA contributes to oncogenesis. The long latency to lymphoma development in our transgenic mice indicates that additional signals are needed for LANA-dependent lymphomagenesis. However, this is not a hit-and-run mechanism, since LANA remained expressed in all tumor cells. In PEL and MCD, secondary signals are thought to be provided by other KSHV oncogenes (41) that are latently expressed in the same cell or act through paracrine signaling. The transgenic mouse model described herein suggests the novel possibility that these secondary signals may be provided by continued antigen exposure or coinfection with other pathogens, as is frequently seen in AIDS patients.

The LANA transgenic mice developed here will allow us to elucidate the molecular mechanism of LANA within the appropriate cellular lineage in vivo, as well as the effect of secondary growth stimuli, due to exogenous or other viral oncogenes such as KSHV's cyclin-D homolog, which as a transgene can cause B and T cell lymphomas in the absence of p53 (42). In addition this model will be useful to test novel therapies against KSHV lymphomas.

\section{Methods}

Transgenic mice. The entire KSHV LANA open reading frame was placed under control of its own transcriptional regulatory region, encompassing 1299 bp upstream of the transcriptional start site to yield plasmid

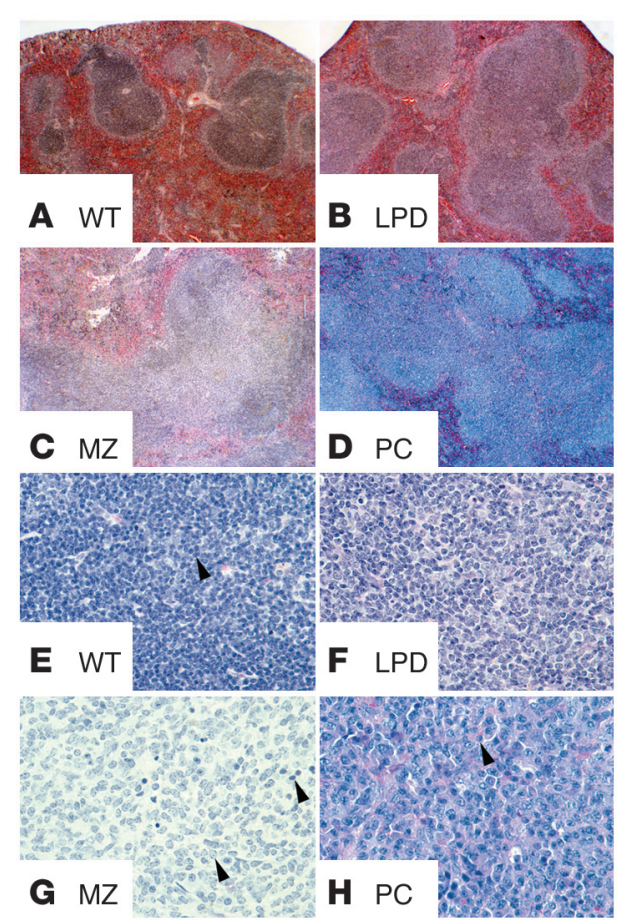

\section{Figure 6}

H\&E stain of wild-type mice and phenotypes in LANA transgenic mice - LPD, MZ lymphoma (MZ), and anaplastic plasmacytoma (PC). Arrowheads indicate individual cells that exemplify the phenotype in panels E, G, and H. Magnification, $\times 100(A-D) ; \times 400(E-H)$. 
A

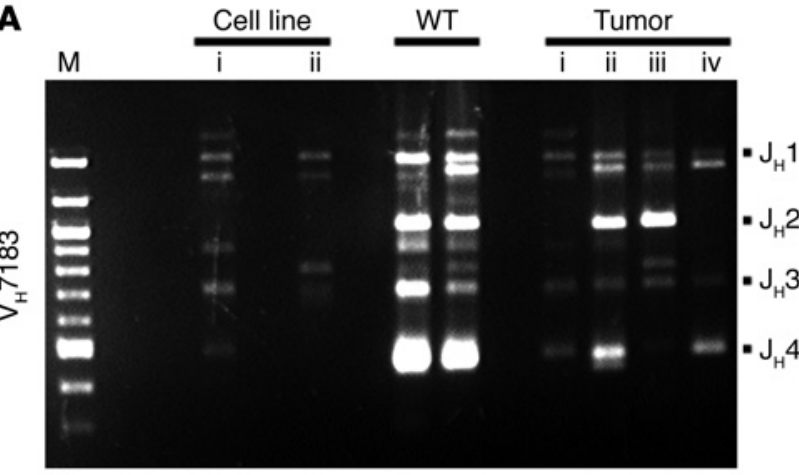

B

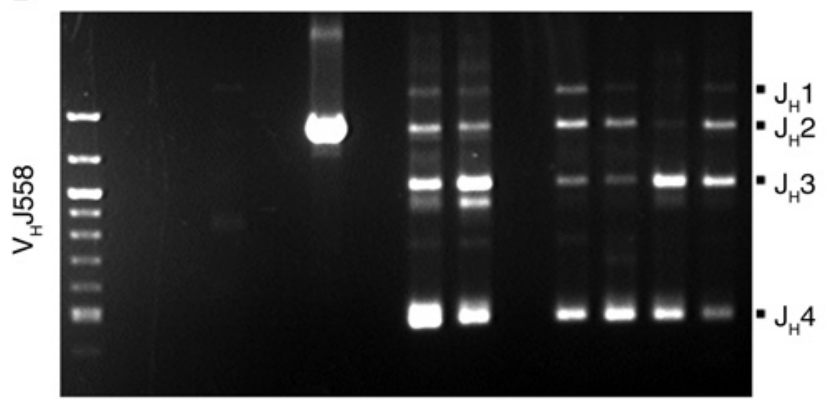

C

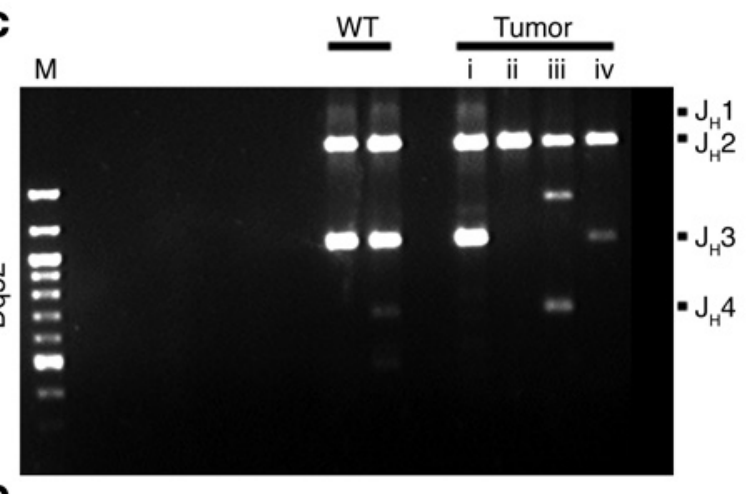

D

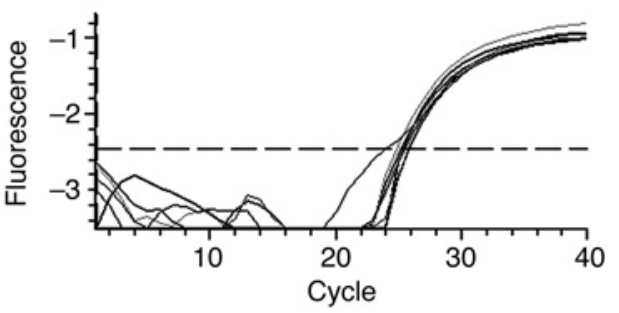

Figure 7

PCR analysis of $V D J_{H}$ and $D J_{H}$ rearrangements in tumors of LANA transgenic mice. Total spleen genomic DNAs were used in the PCR reactions analyzing tumors. (A-C) PCR analysis of total spleen genomic DNAs from 4 LANA transgenic mice with tumors, 2 C57BL/6 mice older than 300 days (WT), and 2 different murine $B$ cell lines resolved on a 1.5\% Tris-acetate-EDTA-agarose gel, used as controls for the clonality. WT lanes

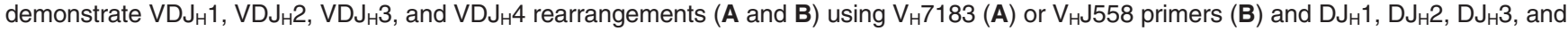
$D J_{H} 4$ rearrangements $(C)$ using Dq52 primers. The primers were designed to amplify all rearrangement products between $J_{H} 4$ and $V_{H} 7183, V_{H} J 558$, or Dq52; 4 different bands would be expected by the combination of each primer set. M, 100-bp DNA Ladder (New England Biolabs Inc.) (D) DNA samples used for the Ig rearrangement PCRs were quantified using real-time quantitative PCR to demonstrate equal total DNA concentrations.

pDD445. As an additional guard against possible epigenetic inactivation of the viral promoter, we included the promoter for the cellular TK gene, which is active in many tissues and has been used previously in transgenic models, downstream of the LANA coding region. Transgenic mice were generated by the National Institute of Child Health and Human Development (NICHD) Transgenic Mouse Development Facility at the University of Alabama at Birmingham. Southern blotting and real-time quantitative RT-PCR were performed as previously described (27). All experiments were approved by the Institutional Animal Care and Use Committees at the University of Oklahoma Health Sciences Center and the University of North Carolina at Chapel Hill.

Immunohistochemistry. Spleens were removed, fixed in $10 \%$ neutral buffered formalin, embedded in paraffin, and processed by routine methods, and $5-\mu \mathrm{m}$ sections were obtained. Dewaxed sections were microwaved in $1 \mathrm{mM}$ EDTA ( $\mathrm{pH}$ 8.0) for PCNA and PNA, RetrievagenA for rat mAb to LANA, or RetrievagenB (BD Biosciences - Pharmingen) for all other antibodies for 15 minutes, cooled, treated with $3 \% \mathrm{H}_{2} \mathrm{O}_{2}$, blocked with $10 \%$ horse serum, and incubated overnight with the appropriate primary antibody. Detection was with VECTASTAIN ABC kit and Vector NovaRed substrate (Vector Laboratories). Slides were counterstained with hematoxylin.

Immunofluorescent staining. Spleens were removed, fixed in 10\% neutral buffered formalin, embedded in paraffin, and processed by routine methods, and $5-\mu \mathrm{m}$ sections were obtained. Dewaxed sections were microwaved in 1 mM EDTA ( $\mathrm{pH} 8.0$ ), blocked with $10 \%$ horse serum, and incubated with FITC-conjugated anti-mouse $\kappa$ or FITC-conjugated anti-mouse $\operatorname{Ig} \lambda 1, \lambda 2$, and $\lambda 3$ light chain for 1 hour.
Flow cytometry. Single-cell suspensions were prepared from spleens by pressing through $100-\mu \mathrm{m}$ cell strainers. Erythrocytes were depleted using buffered ammonium chloride. Cells were stained at $1 \times 10^{8} \mathrm{cells} / \mathrm{ml}$ on ice for 1 hour in the dark, with a cocktail of 3 or 4 antibodies conjugated with PE, FITC, TRI-color (TC), and allophycocyanin (APC) diluted in PBS with 3\% BSA. Flow cytometry analysis was performed with FACScalibur and CellQuest program (CellQuestPro; BD Biosciences). Further analysis was conducted using FlowJo (version 6.1; Tree Star Inc.) and SPSS software (version 11.0; SPSS).

Antibodies. PE-conjugated anti-mouse IgM (clone R6-60.2), PE-conjugated anti-mouse CD21/CD35 (clone 7G6), PE-conjugated anti-mouse CD138 (Syndecan-1), FITC-conjugated anti-mouse IgD (clone 11-26c.2a), and FITC-conjugated anti-mouse $\operatorname{Ig} \lambda 1, \lambda 2$, and $\lambda 3$ light chain (clone R26-46) mAbs were purchased from BD Biosciences; FITC-conjugated anti-mouse MHC class II (clone M5/114.15.2) mAb was from Miltenyi Biotec; PE-conjugated anti-mouse CD27 (clone LG.7F9) mAb was from eBioscience; FITC-conjugated anti-mouse CD38 (clone 90), anti-human CD27 (clone CLB-27/1), anti-mouse $\kappa$ (clone LO-MK), phycoerythrin-conjugated anti-mouse CD23 (clone B3B4), anti-mouse CD40 (clone 3/23), APC-conjugated CD23, TC-conjugated anti-mouse CD19 (clone 6D5), and anti-mouse CD45R (clone RA3-6B2) mAbs were from CALTAG Laboratories; rat mAb to LANA was from Advanced Biotechnologies Inc.; antiPCNA rabbit polyclonal IgG (FL-261) was from Santa Cruz Biotechnology Inc.; Biotinylated Peanut Agglutinin was from Vector Laboratories.

Immunization. Transgenic and littermate control mice (29-34 weeks old) were immunized i.p. with $100 \mu \mathrm{g} \mathrm{NP}(30)-\mathrm{KLH}$ alum precipitated (Biosearch Technologies Inc.) in $0.2 \mathrm{ml}$ saline or $0.2 \mathrm{ml}$ saline (mock). 
Detection of Ig gene rearrangements. DNA was isolated from spleens using Wizard SV Genomic DNA kit (Promega). PCR was set up in a volume of $20 \mu \mathrm{l}$ with $2 \times$ ImmoMix (Bioline), $1.7 \mathrm{ng}$ genomic DNA, and $250 \mathrm{nmol}$ primers. PCR reactions were preheated for 5 minutes at $92^{\circ} \mathrm{C}$, followed by 40 cycles of 30 seconds at $95^{\circ} \mathrm{C}, 30$ seconds at $56^{\circ} \mathrm{C}$, and 3 minutes at $72^{\circ} \mathrm{C}$. Primers for detection of $\mathrm{Ig}$ rearrangements were described previously (43-46). The primers were designed to amplify all rearrangement products between $\mathrm{J}_{\mathrm{H}} 4$ and $\mathrm{V}_{\mathrm{H}} 7183, \mathrm{~V}_{\mathrm{H}} 558$, or Dq52; if $\mathrm{D}_{\mathrm{H}} \mathrm{J}_{\mathrm{H}}$ and $\mathrm{V}_{\mathrm{H}} \mathrm{D}_{\mathrm{H}} \mathrm{J}_{\mathrm{H}}$ rearrangements had occurred, 4 bands were observed depending on the $\mathrm{J}_{\mathrm{H}}$ element $\left(\mathrm{J}_{\mathrm{H}} 1, \mathrm{~J}_{\mathrm{H}} 2, \mathrm{~J}_{\mathrm{H}} 3\right.$, or $\left.\mathrm{J}_{\mathrm{H}} 4\right)$ that was used. DNA Ladder (100 bp; New England Biolabs Inc.) was used to determine the size of PCR products. Primers used were: Figure 7A, 5'-GCAGCTGGTGGAGTCTGG-3' and 5'-CTCTCAGCCGGCTCCCTCAGGG-3'; Figure 7B, 5'-CAGGTCCAACTGCAGCAG- $3^{\prime}$ and $5^{\prime}$-CTCTCAGCCGGCTCCCTCAGGG-3'; Figure 7C, 5'-GCGGAGCACCACAGTGCAACTGGGAC-3' and 5'-AAAGACCTGCAGAGGCCATTCTTACC-3'; and Figure 7D, 5'-CACGTGGGCTCCAGCATT- $3^{\prime}$ and $5^{\prime}$-TCACCAGTCATTTCTGCCTTTG- $3^{\prime}$.

RNA isolation and RT. RNA was isolated by using TRIAzol (SigmaAldrich) according to the supplier's protocol. poly(A) mRNA was prepared by using dT-beads (QIAGEN), and $500 \mathrm{ng}$ of mRNA was reverse transcribed in a $20-\mu 1$ reaction with $100 \mathrm{U}$ SuperscripII (Invitrogen Corp.), $2 \mathrm{mM}$ deoxynucleoside triphosphates, $2.5 \mathrm{mM} \mathrm{MgCl}_{2}, 1 \mathrm{U}$ RNasin (all from Applied Biosystems), and $0.5 \mu \mathrm{g}$ random hexanucleotide primers (Amersham Pharmacia Biotech) according to our previously published protocol (47). Quantitative PCR was conducted according to our published procedures $(27,47)$ using $2 \times$ SYBR PCR mix (Applied Biosystems).

\section{Acknowledgments}

The authors thank Rebecca Hines-Boykin for invaluable technical help; Barbara Vilen for cell lines; Stephen Clarke, Michelle Staudt, James Papin, and Blossom Damania for critical reading and discussion; and Ethel Cesarman and Amy Chadburn for lymphoma evaluation. F.D. Fakhari conducted this work as part of her thesis for the Graduate Program in Microbiology and Immunology at the University of Oklahoma Health Sciences Center. Supported by the Oklahoma Center for the Advancement of Science (grant HR03-0028), the UNC Lineberger Comprehensive Cancer Center, the NIH (grant CA109232), and the Leukemia \& Lymphoma Society.

Received for publication July 7, 2005, and accepted in revised form January 3, 2006.

Address correspondence to: Dirk P. Dittmer, Department of Microbiology and Immunology, 804 Mary Ellen Jones, CB\#7290, University of North Carolina, Chapel Hill, North Carolina 275997290, USA. Phone: (919) 966-7960; Fax: (919) 962-8103; E-mail: ddittmer@med.unc.edu.
1. Goedert, J.J. 2000. Infectious causes of cancer. Humana Press. Totowa, New Jersey, USA. 489 pp.

2. Ablashi, D.V., Chatlynne, L.G., Whitman, J.E., Jr., and Cesarman, E. 2002. Spectrum of Kaposi's sarcoma-associated herpesvirus, or human herpesvirus 8, diseases. Clin. Microbiol. Rev. 15:439-464.

3. Carbone, A., et al. 2001. Expression profile of MUM1/IRF4, BCL-6, and CD138/syndecan-1 defines novel histogenetic subsets of human immunodeficiency virus-related lymphomas. Blood. 97:744-751.

4. Klein, U., et al. 2003. Gene expression profile analysis of AIDS-related primary effusion lymphoma (PEL) suggests a plasmablastic derivation and identifies PEL-specific transcripts. Blood. 101:4115-4121.

5. Carbone, A., et al. 2005. Kaposi's sarcoma-associated herpesvirus/human herpesvirus type 8-positive solid lymphomas: a tissue-based variant of primary effusion lymphoma. J. Mol. Diagn. 7:17-27.

6. Deloose, S.T., et al. 2005. High incidence of Kaposi sarcoma-associated herpesvirus infection in HIVrelated solid immunoblastic/plasmablastic diffuse large B-cell lymphoma. Leukemia. 19:851-855.

7. Katano, H., et al. 2000. Human herpesvirus 8associated solid lymphomas that occur in AIDS patients take anaplastic large cell morphology. Mod. Pathol. 13:77-85.

8. Chadburn, A., et al. 2004. KSHV-positive solid lymphomas represent an extra-cavitary variant of primary effusion lymphoma. Am. J. Surg. Pathol. 28:1401-1416

9. Jenner, R.G., et al. 2003. Kaposi's sarcoma-associated herpesvirus-infected primary effusion lymphoma has a plasma cell gene expression profile. Proc. Natl. Acad. Sci. U. S. A. 100:10399-10404.

10. Fan, W., et al. 2005. Distinct subsets of primary effusion lymphoma can be identified based on their cellular gene expression profile and viral association. J. Virol. 79:1244-1251.

11. Fakhari, F.D., and Dittmer, D.P. 2002. Charting latency transcripts in Kaposi's sarcoma-associated herpesvirus by whole-genome real-time quantitative PCR. J. Virol. 76:6213-6223.

12. Staudt, M.R., et al. 2004. The tumor microenvironment controls primary effusion lymphoma growth in vivo. Cancer Res. 64:4790-4799.

13. Dittmer, D.P. 2003. Transcription profile of Kaposi's sarcoma-associated herpesvirus in primary Kaposi's sarcoma lesions as determined by realtime PCR arrays. Cancer Res. 63:2010-2015.

14. Dittmer, D., et al. 1998. A cluster of latently expressed genes in Kaposi's sarcoma-associated herpesvirus. J. Virol. 72:8309-8315.

15. Dupin, N., et al. 1999. Distribution of human herpesvirus-8 latently infected cells in Kaposi's sarcoma, multicentric Castleman disease, and primary effusion lymphoma. Proc. Natl. Acad. Sci. U. S. A. 96:4546-4551.

16. Ballestas, M.E., Chatis, P.A., and Kaye, K.M. 1999. Efficient persistence of extrachromosomal KSHV DNA mediated by latency- associated nuclear antigen. Science. 284:641-644.

17. Cotter, M.A., 2nd, and Robertson, E.S. 1999. The latency-associated nuclear antigen tethers the Kaposi's sarcoma- associated herpesvirus genome to host chromosomes in body cavity-based lymphoma cells. Virology. 264:254-264.

18. Garber, A.C., Shu, M.A., Hu, J., and Renne, R. 2001. DNA binding and modulation of gene expression by the latency-associated nuclear antigen of Kaposi's sarcoma-associated herpesvirus. J. Virol. 75:7882-7892.

19. Friborg, J., Jr., Kong, W., Hottiger, M.O., and Nabel, G.J. 1999. p53 inhibition by the LANA protein of KSHV protects against cell death. Nature. 402:889-894.

20. Fujimuro, M., et al. 2003. A novel viral mechanism for dysregulation of beta-catenin in Kaposi's sarcoma-associated herpesvirus latency. Nat. Med. 9:300-306.

21. Radkov, S.T., Kellam, P., and Boshoff, C. 2000. The latent nuclear antigen of Kaposi sarcoma-associated herpesvirus targets the retinoblastoma-E2F pathway and with the oncogene Hras transforms primary rat cells. Nat. Med. 6:1121-1127.

22. Jeong, J., Papin, J., and Dittmer, D. 2001. Differential regulation of the overlapping Kaposi's sarcoma-associated herpesvirus vGCR (orf74) and LANA (orf73) promoters. J. Virol. 75:1798-1807.

23. Renne, R., et al. 2001. Modulation of cellular and viral gene expression by the latency-associated nuclear antigen of Kaposi's sarcoma-associated herpesvirus. J. Virol. 75:458-468.

24. Knight, J.S., Cotter, M.A., 2nd, and Robertson, E.S. 2001. The latency-associated nuclear antigen of Kaposi's sarcoma-associated herpesvirus transactivates the telomerase reverse transcriptase promoter. J. Biol. Chem. 276:22971-22978.

25. An, F.Q., et al. 2005. The latency-associated nuclear antigen of Kaposi's sarcoma-associated herpesvirus modulates cellular gene expression and protects lymphoid cells from $\mathrm{p} 16 \mathrm{INK} 4 \mathrm{~A}$-induced cell cycle arrest. J. Biol. Chem. 280:3862-3874.

26. Chen, J., et al. 2001. Activation of latent Kaposi's sarcoma-associated herpesvirus by demethylation of the promoter of the lytic transactivator. Proc. Natl. Acad. Sci. U. S. A. 98:4119-4124.

27. Jeong, J.H., Hines-Boykin, R., Ash, J.D., and Dittmer, D.P. 2002. Tissue specificity of the Kaposi's sarcoma-associated herpesvirus latent nuclear antigen (LANA/orf73) promoter in transgenic mice. J. Virol. 76:11024-11032.

28. Morse, H.C., 3rd, et al. 2002. Bethesda proposals for classification of lymphoid neoplasms in mice. Blood. 100:246-258.

29. Lalor, P.A., Nossal, G.J., Sanderson, R.D., and McHeyzer-Williams, M.G. 1992. Functional and molecular characterization of single (4-hydroxy3-nitrophenyl)acetyl (NP)-specific, IgG1+ B cells from antibody-secreting and memory $\mathrm{B}$ cell pathways in the C57BL/6 immune response to NP. Eur. J. Immunol. 22:3001-3011.

30. de Sanjose, S., et al. 2004. Risk of malignant lymphoma associated with human herpesvirus-8: a casecontrol study in Spain. Br. J. Cancer. 90:2145-2148.

31. Du, M.Q., et al. 2001. Kaposi sarcoma-associated herpesvirus infects monotypic (IgM lambda) but polyclonal naive $\mathrm{B}$ cells in Castleman disease and associated lymphoproliferative disorders. Blood. 97:2130-2136.

32. Teicher, B. 2002. Tumor models in cancer research. Humana Press. Totowa, New Jersey, USA. 690 pp.

33. Decker, L.L., et al. 1996. The Kaposi sarcoma-associated herpesvirus (KSHV) is present as an intact latent genome in KS tissue but replicates in the peripheral blood mononuclear cells of KS patients. J. Exp. Med. 184:283-288. 
34. Bechtel, J.T., Liang, Y., Hvidding, J., and Ganem, D. 2003. Host range of Kaposi's sarcoma-associated herpesvirus in cultured cells. J. Virol. 77:6474-6481.

35. Dittmer, D., et al. 1999. Experimental transmission of Kaposi's sarcoma-associated herpesvirus (KSHV/HHV-8) to SCID-hu Thy/Liv Mice. J. Exp. Med. 190:1857-1868.

36. Whitby, D., et al. 1995. Detection of Kaposi sarcoma associated herpesvirus in peripheral blood of HIV-infected individuals and progression to Kaposi's sarcoma. Lancet. 346:799-802.

37. Martin, J.N., et al. 1998. Sexual transmission and the natural history of human herpesvirus 8 infection. N. Engl. J. Med. 338:948-954.

38. Forster, I., and Rajewsky, K. 1990. The bulk of the peripheral B-cell pool in mice is stable and not rapidly renewed from the bone marrow. Proc. Natl. Acad. Sci. U. S. A. 87:4781-4784.
39. Grundhoff, A., and Ganem, D. 2004. Inefficient establishment of KSHV latency suggests an additional role for continued lytic replication in Kaposi sarcoma pathogenesis. J. Clin. Invest. 113:124-136. doi:10.1172/JCI200417803.

40. Cattoretti, G., et al. 2005. Deregulated BCL6 expression recapitulates the pathogenesis of human diffuse large B cell lymphomas in mice. Cancer Cell. 7:445-455.

41. Montaner, S., et al. 2003. Endothelial infection with KSHV genes in vivo reveals that vGPCR initiates Kaposi's sarcomagenesis and can promote the tumorigenic potential of viral latent genes. Cancer Cell. 3:23-36.

42. Verschuren, E.W., et al. 2004. The role of p53 in suppression of KSHV cyclin-induced lymphomagenesis. Cancer Res. 64:581-589.

43. Wei, C., Zeff, R., and Goldschneider, I. 2000. Murine
pro-B cells require IL-7 and its receptor complex to up-regulate IL-7R alpha, terminal deoxynucleotidyltransferase, and c mu expression. J. Immunol. 164:1961-1970.

44. Chang, Y., Paige, C.J., and Wu, G.E. 1992. Enumeration and characterization of DJH structures in mouse fetal liver. EMBO J. 11:1891-1899.

45. Campbell, M.R., Nation, P.N., and Andrew, S.E. 2005. A lack of DNA mismatch repair on an athymic murine background predisposes to hematologic malignancy. Cancer Res. 65:2626-2635.

46. Fujimura, S., et al. 2005. Increased expression of germinal center-associated nuclear protein RNAprimase is associated with lymphomagenesis. Cancer Res. 65:5925-5934.

47. Papin, J., Vahrson, W., Hines-Boykin, R., and Dittmer, D.P. 2004. Real-time quantitative PCR analysis of viral transcription. Methods Mol. Biol. 292:449-480. 